\title{
Editorial
}

\section{Más allá de la enfermedad sin pulsos}

En este número de la revista Hernández-de Mezerville y colaboradores, y Vargas-Sanabria y Valerio-Hernández, nos sorprenden con la descripción de dos casos de presentaciones diferentes de una forma de vasculitis tan interesante como poco conocida: la Arteritis de Takayasu (AT).

Las vasculitis se definen sencillamente como enfermedades donde existe inflamación de los vasos sanguíneos y deben sospecharse en pacientes con múltiples manifestaciones inusuales asociadas a signos de inflamación, que casi siempre, al inicio de su padecimiento, no parecieran encajar en ningún diagnóstico específico.

La mayoría de las vasculitis pueden afectar tanto adultos como niños, sin embargo, la frecuencia de las mismas es radicalmente diversa en los dos grupos de edad. Así, por ejemplo, la Enfermedad de Kawasaki es mucho más común en la edad pediátrica, en tanto la Arteritis Temporal es una enfermedad típicamente del adulto. Otras vasculitis como la Poliarteritis y la Granulomatosis de Wegener tienen características etiológicas, clínicas y pronósticas diferentes en los niños que en los adultos. Por lo anterior, en la actualidad existe consenso internacional respecto al hecho de que es inapropiado aplicar las clasificaciones de vasculitis del adulto en el ámbito pediátrico. Existe ya una propuesta de clasificación pediátrica elaborada por un grupo de la Sociedad Europea de Reumatología (PreS, por sus siglas en inglés), el Comité de Reumatología Pediátrica de EULAR (Liga Europea contra la Artritis y Reumatismo), el Colegio Americano de Reumatología (ACR, por sus siglas en inglés) y la Sociedad Europea de Nefrología Pediátrica. ${ }^{1}$ En general, las clasificaciones propuestas tanto para niños como para adultos, subdividen las vasculitis según el calibre de los vasos involucrados y clasifican la enfermedad de pequeños vasos en granulomatosa y no granulomatosa.

La AT es la típica vasculitis de grandes vasos que involucra principalmente la aorta, sus ramas principales y arterias coronarias y pulmonares. Además de síntomas constitucionales como fiebre prolongada, pérdida de peso, dolor músculo-esquelético y ataque al estado general, AT causa otras manifestaciones clínicas como claudicación en los brazos, disminución en los pulsos arteriales, dolor a la palpación carotídea, pérdida visual, accidente vascular cerebral, regurgitación aórtica, hipertensión e insuficiencia cardiaca congestiva.

Como se ilustra en el caso reportado en este número de la revista por Bravo-Rojas y colaboradores, la presentación clínica puede ser insidiosa y el diagnóstico es con frecuencia retardado. En la actualidad se trabaja en la identificación de factores pronósticos individuales relacionados con el curso y desenlace de la enfermedad. La presencia de ciertos antígenos de HLA, así como la velocidad de eritrosedimentación parecieran ser predictores importantes. ${ }^{2}$

Clásicamente se ha descrito un patrón trifásico de enfermedad con una fase temprana (preausencia de pulsos) dominada por los síntomas constitucionales, seguida por la fase de inflamación vascular activa en que se evidencia dolor en las áreas afectadas y signos de insuficiencia vascular, finalizando con la etapa donde aparecen las lesiones vasculares fibróticas y estenóticas. Sin embargo, el hallazgo de evidencia histológica de inflamación en $40 \%$ de pacientes considerados con enfermedad inactiva ha servido de fundamento para cuestionar el dogma del patrón trifásico y ha impulsado en la actualidad la búsqueda de criterios para la evaluación de la actividad de la enfermedad. ${ }^{3}$

La AT es epidemiológicamente conocida por su predominio en pacientes femeninas y por marcadas diferencias étnicas, con altas prevalencias en naciones asiáticas y bajas prevalencias en poblaciones caucásicas. Es interesante destacar que grupos importantes de casos han sido reportados en sitios de Latinoamérica como Perú, Colombia, México y Brasil ${ }^{4}$.

Acta Médica Costarricense, C2007 Colegio de Médicos y Cirujanos 
Como es evidente, desde la descripción inicial de esta condición mórbida, reportada en 1830 por Yamamoto en Japón, ${ }^{5}$ y la caracterización de algunos de los casos como "enfermedad sin pulsos", muchos aspectos han cambiado. Fundamentalmente, los grandes progresos en tecnología médica han hecho posible que la AT se diagnostique antes de que se convierta en una "enfermedad sin pulsos", por lo que esta denominación no pareciera ser la adecuada en nuestros tiempos. Los análisis angiográficos no invasivos por tomografía computarizada y/o la angiografía por resonancia magnética han hecho más fácil la detección de la enfermedad, el diagnóstico temprano, el tratamiento oportuno y, nos han permitido conocer un espectro más amplio de las manifestaciones clínicas que presenta. ${ }^{6}$

La causa de la AT continúa siendo desconocida. Se considera una enfermedad multifactorial, en la cual las fuertes diferencias étnicas en sus manifestaciones clínicas, así como el reporte de múltiples casos en gemelos idénticos, indican un importante papel de los factores genéticos en su patogénesis ${ }^{4,7}$. Los factores que estimulan al sistema HLA y el papel de diversos elementos accesorios en sus manifestaciones clínicas y comportamiento epidemiológico, son interesantes preguntas para resolver en el futuro. La implementación de registros internacionales de casos de patologías poco frecuentes como ésta, es probablemente un paso fundamental para dar respuesta a las interrogantes pendientes.

Olga Arguedas-Arguedas

Universidad de Costa Rica. Hospital Nacional de Niños

"Dr. Carlos Sáenz Herrera". Caja Costarricense de

Seguro Social.

\section{Referencias}

1. Ozen S, Ruperto N, Dillon MJ, Bagga A, Barron K, Davin JC, Kawasaki T, Lindsley C, Petty RE, Prieur AM, Ravelli A, Woo P. EULAR/ PreS endorsed consensus criteria* for the classification of childhood vasculitides. Ann Rheum Dis. 2006; 65:936-941

2. Vanoli M, Bacchiani G, Origgi L, Scorza R. Takayasu Arteritis: a changing disease. J Nephrol 2001; 14:497- 505

3. Lagneau P, Michel JB, Vuong PN. Surgical treatment of Takayasu's disease. Ann Surg 1986; 205: 157-66

4. Numano F. Differences in clinical presentation and outcome in different countries for Takayasu Arteritis. Curr Opin Rheumatol. 1997; 9:12-15

5. Numano F, Kakuta T. Takayasu Arteritis-Five doctors in the history of Takayasu Arteritis. Int J cardiol. 1996; 54: suppl: 1-10

6. Yamada I, Numano F, Susuki S. Takayasu Arteritis, Evaluation with MR imaging. Radiology. 1993; 188: 89-94

7. Numano F, Kobayashi Y. Takayasu Arteritis-beyond PulselessnessIntern Med 1999; 38:226-32 